\title{
Estimating the Prevalence and the Determinants of Polypharmacy Using Data from a Health Administrative Database: A Comparison of Results Obtained Employing Different Algorithms
}

\author{
Valeria Fano ${ }^{1 *}$, Francesco Chini ${ }^{2}$, Patrizio Pezzotti ${ }^{3}$ and Katia Bontempi ${ }^{1}$ \\ ${ }^{1}$ Local Health Authority RMD, Rome, Italy \\ ${ }^{2}$ Agency for Public Health of Lazio Region, Rome, Italy \\ ${ }^{3}$ Istituto Superiore di Sanità, Rome, Italy
}

\begin{abstract}
Background: Few studies have been carried out to estimate the prevalence of polypharmacy in the general population using administrative databases. Different methods and definitions have been proposed, but no comparisons have been provided. The aim of this study is to estimate the prevalence and the determinants of polypharmacy in Rome (Italy).
\end{abstract}

Methods: Adults $(35+; n=331,923)$ residing in 2008 in the Local Health Authority 'Roma D' (southern part of Rome) were included; prescriptions (years 2009-12) were retrieved from a database which collects information on all drugs prescribed. Three algorithms were defined: (1) the number of different drugs prescribed for at least 60 days per year; the number of different drugs prescribed for at least 60 days per quarter per year, using 90-days-fixed- (2), and -mobile-windows (3). Determinants of polypharmacy based on patients' and general practitioner' (GP) characteristics were investigated using multilevel logistic regression models.

Results: The prevalence of major polypharmacy (>5 drugs) ranged between 6 and 10\%, depending on the algorithm used, yielding estimates similar to those in the existing literature. Algorithm 1 provided higher estimates than Algorithm 2 and 3; a temporal increase of about 3\% for each algorithm was observed as well. Multilevel models showed that polypharmacy was more frequent among women, Italian-born-subjects, elderly, patients with $\geq 3$ comorbidities, and subjects living in disadvantaged areas. No particular differences were detected by GPs' characteristics.

Conclusions: Polypharmacy is an emerging public health issue with increasing prevalence. Prevalence estimates were sensitive to the algorithm used. Major polypharmacy was principally related to age and comorbidities, but other patient's characteristics may play a role as well.

Keywords: Polypharmacy; Drugs; Population; Adverse drug events

\section{Introduction}

Polypharmacy is an emerging public health issue with increasing prevalence in developed countries. The term "polypharmacy" has been defined in several ways: the concomitant use of multiple drugs; the use of more drugs than is clinically appropriated; the use of potentially inappropriate medications; and the duplication of medication [1]. The increase in polypharmacy has been associated with an increased risk of adverse drug events, both due to the high risk of exposure to adverse drug-to-drug interaction, and to the decrease in adherence to therapies when the number of drugs increases [2-4]. Most of the studies investigating polypharmacy have focused on the elderly population, given the higher prevalence of concomitant diseases, resulting in the administration of multiple therapies. Although there is agreement on the adverse health outcomes associated with polypharmacy, the number of simultaneous medications that should be considered as a threshold for the definition of polypharmacy is still undefined. Different methods of identifying cases of polypharmacy were proposed, but no comparisons were provided so far.

Regarding the definition of "concomitant use" of drugs, some investigators have defined polypharmacy as the concomitant use of four or more medications [5], others as five or more [6-9], and others as the long-term simultaneous use of two or more medications [10]. Recent studies focus the definition of polypharmacy on the specific context in which the use of a drug combination is associated with a high risk of adverse effects [11,12]. A recent study [13] identifies the concomitant use of five or more drugs as the optimal cut-off to estimate the drugrelated adverse effects of frailty, disability, falls and mortality in the elderly over 70 years of age.

To date, only few studies have been carried out to estimate the prevalence of polypharmacy in the general population using administrative databases $[8,9,14]$; all methods previously used estimated yearly prevalence by means of fixed time windows such as one day (point prevalence) $[5,7,12]$ or one trimester $[8,10,14]$; one study defined 60 days of therapy as the minimum duration of therapy to be considered in the case detection [10]. Methods based on fixed time windows might underestimate the prevalence of polytherapy, failing to detect treatments beginning in a time window and ending in a different one; on the other hand, yearly prevalence could overestimate

*Corresponding author: Dr. Valeria Fano, Local Health Authority RMD, Rome Italy, Tel: +390656487200; Fax: +390656487205; E-mail: valeria.fano@asIromad.it

Received March 21, 2014; Accepted April 29, 2014; Published May 01, 2014

Citation: Fano V, Chini F, Pezzotti P, Bontempi K (2014) Estimating the Prevalence and the Determinants of Polypharmacy Using Data from a Health Administrative Database: A Comparison of Results Obtained Employing Different Algorithms. Adv Pharmacoepidemiol Drug Saf 3: 151. doi:10.4172/2167-1052.1000151

Copyright: (c 2014 Fano V, et al. This is an open-access article distributed unde the terms of the Creative Commons Attribution License, which permits unrestricted use, distribution, and reproduction in any medium, provided the original author and source are credited. 
Citation: Fano V, Chini F, Pezzotti P, Bontempi K (2014) Estimating the Prevalence and the Determinants of Polypharmacy Using Data from a Health Administrative Database: A Comparison of Results Obtained Employing Different Algorithms. Adv Pharmacoepidemiol Drug Saf 3: 151. doi:10.4172/2167-1052.1000151

Page 2 of 7

the real drug consumption as it is usually based on a minimum length of therapy (e.g. 60 days) and could include short occasional use which summed up over the year could match the polytherapy definition. In order to minimize both type of error, we propose a new method based on a mobile time window.

The aim of this study is to estimate the prevalence and the determinants of polypharmacy in a population-based cohort of adult individuals resident in Rome, Italy. A prescription database is used; a new algorithm is proposed and compared with the existing ones.

\section{Methods}

\section{Study population}

The study base included all residents in the Local Health Authority "Roma D", which comprises the southern part of the municipality of Rome $(2,844,821$ inhabitants) and the municipality of Fiumicino (66,510 inhabitants), for a total of 575,912 inhabitants. Residents above 34 years of age on January 1, 2008 were included in the study.

The presence of one or more chronic and/or severe illnesses at baseline as well as census tract information on socioeconomic status (i.e., deprivation index) were available for this data set until December 31, 2010, as described elsewhere [15]; follow-up was completed until December 31, 2012 through record linkage with hospital admissions and mortality records. For each year, the Charlson Comorbidity Index was computed in order to estimate patient comorbidities (i.e. diseases), through record linkage with acute hospital admissions for the main chronic causes of hospitalization [16]. The Charlson index was computed for the entire period 2008-2012 considering the maximum number of comorbidities resulting from year-specific Charlson's indexes, each referred to previous 5 years' hospital admissions.

All prescriptions filled by the resident population during the period 2008-2012 were retrieved from the Drug Prescription Registry for the Lazio region, which collects all drug prescriptions purchased in the Region and contains information on all drugs prescribed by general practitioners (GP) and public ambulatories, including patient demographic characteristics, code of the Anatomical Therapeutic Chemical Classification System (ATC), number of packages, the date of the prescription, and the standard Defined Daily Doses (DDD) [17]. Record linkage between the population database and all other health sources was performed through a deterministic linkage procedure using the tax code as unique identifier. GPs characteristics were retrieved from the Regional Informative System of GPs $(N=5,005)$ which contains the following information: age; gender; whether they are General Practitioners or Pediatricians; how long they have practiced medicine; and the number of patients in their practice. Databases were linked through deterministic record linkage using physician's codes as unique identifiers.

\section{Definition of polypharmacy and Statistical analyses}

Polypharmacy was defined as "minor" (2-3 different drugs), "moderate" (4-5 different drugs), "major" ( $\geq 6$ different drugs) on the basis of the number of filled prescriptions, each with at least 60 days of therapy in a year for each specific ATC code. Given that for some therapeutic schemes it is possible to switch to a different drug within the same class, polypharmacy was also calculated using the first 4-digits

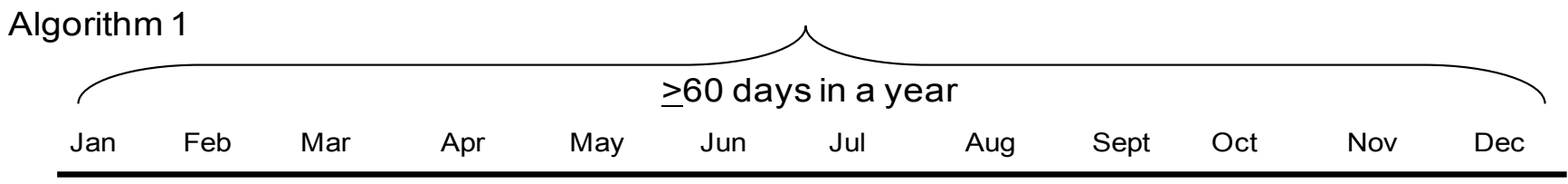

Algorithm 2
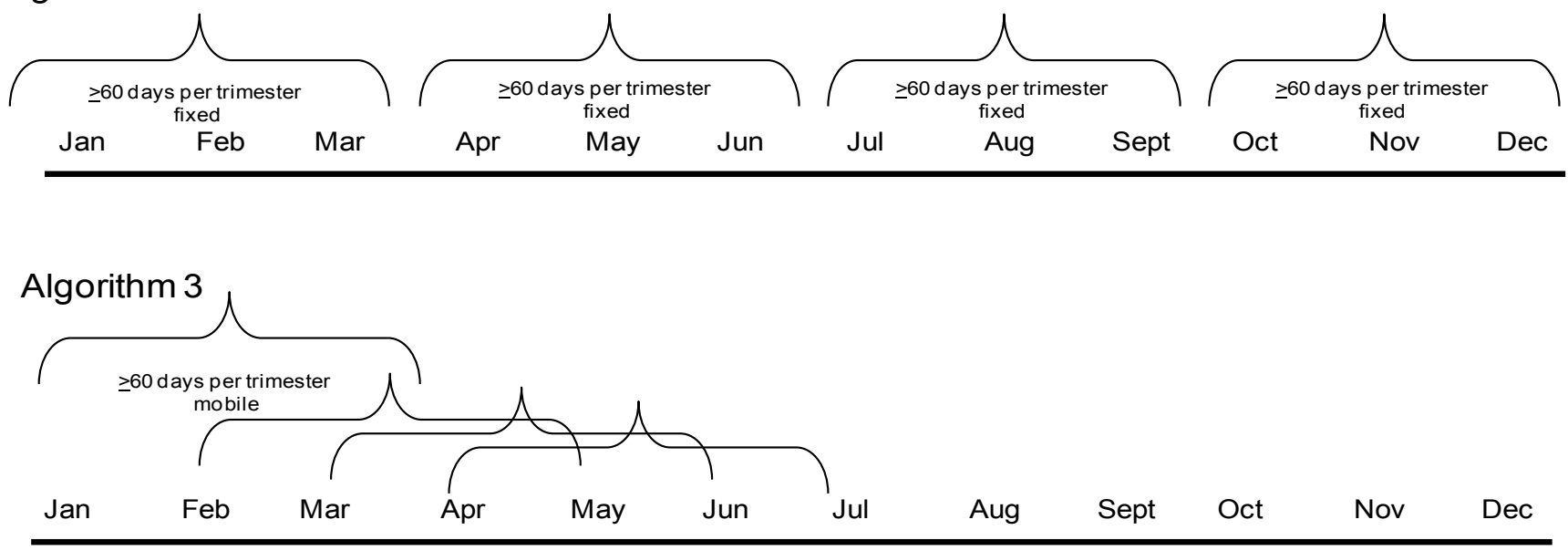

Algorithm 1: number of drugs (different ATC class, 4 digits) prescribed for at least 60 days of therapy per year; Algorithm 2: number of drugs (different ATC class, 4 digits) prescribed for at least 60 days per quarter per year (fixed time windows); Algorithm 3: number of drugs (different ATC class, 4 digits) prescribed for at least 60 days per quarter per year (mobile window).

Figure 1: Methods used to estimate polypharmacy among adult residents in the Local Health unit Roma D. 
Citation: Fano V, Chini F, Pezzotti P, Bontempi K (2014) Estimating the Prevalence and the Determinants of Polypharmacy Using Data from a Health Administrative Database: A Comparison of Results Obtained Employing Different Algorithms. Adv Pharmacoepidemiol Drug Saf 3: 151. doi:10.4172/2167-1052.1000151

Page 3 of 7

\begin{tabular}{|c|c|c|c|}
\hline & & $\begin{array}{c}\mathrm{N} \\
\text { (total }=331,923)\end{array}$ & $\%$ \\
\hline \multirow[t]{2}{*}{ Gender } & Women & 180,761 & 54.5 \\
\hline & Men & 151,162 & 45.5 \\
\hline \multirow[t]{3}{*}{ Age (years) } & $35-49$ & 134,543 & 40.5 \\
\hline & $50-54$ & 93,603 & 28.2 \\
\hline & $65+$ & 103,777 & 31.3 \\
\hline \multirow[t]{2}{*}{ Nationality } & Italian & 306,123 & 92.7 \\
\hline & Non-Italian & 24,060 & 7.3 \\
\hline \multirow[t]{3}{*}{ Deprivation index } & Low & 77,597 & 24.0 \\
\hline & Medium & 181,863 & 56.3 \\
\hline & High & 63,850 & 19.7 \\
\hline \multirow[t]{3}{*}{ Number of comorbidities } & None & 273,966 & 82.5 \\
\hline & $1-2$ & 48,790 & 14.7 \\
\hline & $3+$ & 9,167 & 2.8 \\
\hline \multirow[t]{2}{*}{ Vital status (end of follow-up) } & Alive & 312,753 & 94.2 \\
\hline & Dead & 19,170 & 5.8 \\
\hline
\end{tabular}

Totals may vary due to missing information; Nationality: Nationality at birth; Vital status: vital status at 31.12.2012; Deprivation index: composite area-based deprivation index of census tract of the area of residence; Number of comorbidities: maximum number of comorbidities resulting from year-specific Charlson's indexes referred to previous 5 years hospital admissions.

Table 1: Demographic characteristics of the study population. Adults aged $>35$ years resident in the Local Health Authority Roma D, Italy, at 1.1 .2008 .
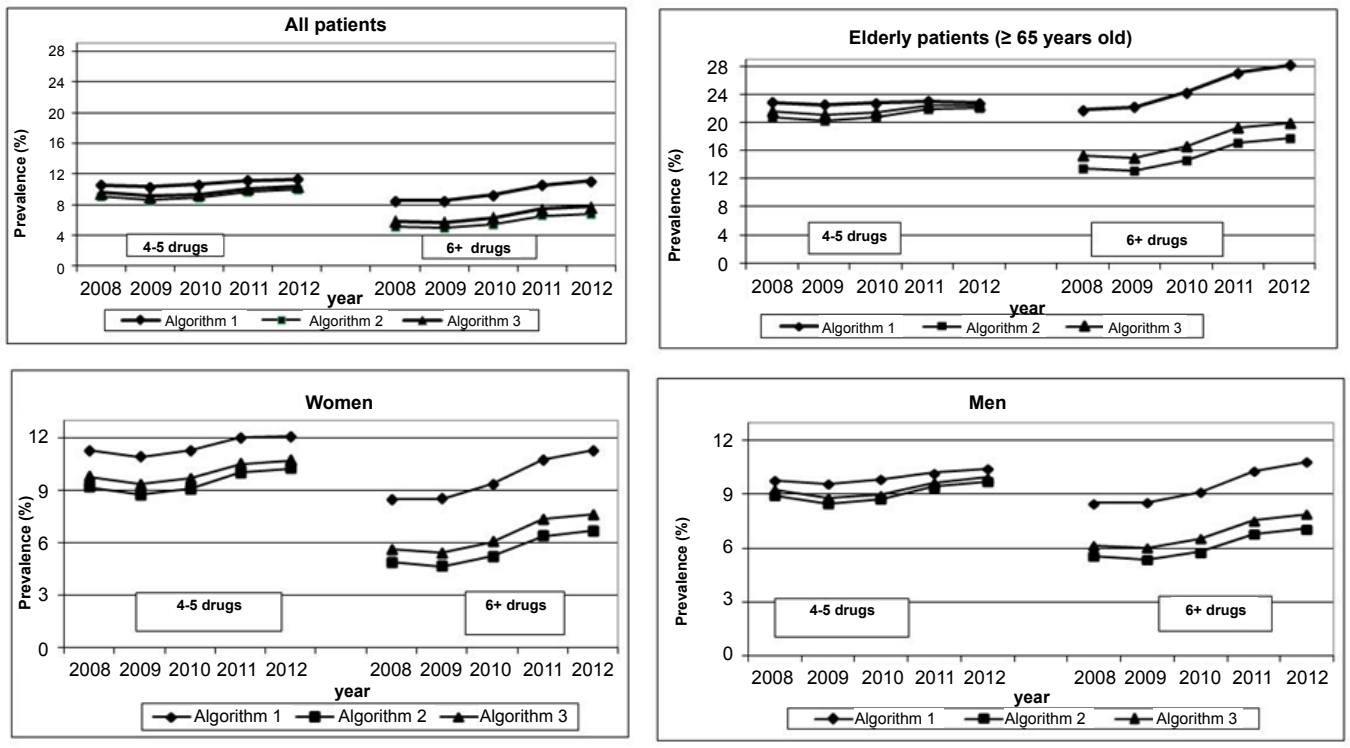

tpolypharmacy defined using up to 4-digits ATC for all algorithm definition

Figure 2: Prevalence of polypharmacy ${ }^{\dagger}$ by calendar year and algorithm definition among different gender and age groups.

of the ATC code instead of the entire 7-digits code.

Three different algorithms were considered to calculate polypharmacy (Figure 1); for each prescription, the length of treatment was computed using the DDDs beginning from the date of prescription. We initially calculated the number of drugs belonging to different ATC classes (4-digits codes) which were prescribed for at least 60 days (Algorithm 1). We then considered the number of different drugs which were prescribed for at least 60 days per quarter per year (i.e., fixed time windows from January to March, and so on; Algorithm 2). Finally, a 60 days-mobile-window was used (i.e., from January to March, from February to April, and so on; Algorithm 3). The use of 60 days as the duration of treatment in each time window was chosen according to standards in the literature [10].
Annual prevalences of drug use were calculated; in order to take into account for the yearly variations in the population size, midyear population was considered for each year of the follow-up period, i.e. residents and alive at June 30 of each year. All analyses were stratified by gender and age class (above/below 65 years).

Finally, the relationship of polypharmacy with several characteristics of the subjects (i.e., gender, age, deprivation index, comorbidities, nationality) and of the GPs (i.e., age, gender, and number of patients) were evaluated using multilevel logistic regression models by grouping patients according to their GP and adjusting for all other variables. Different outcomes for each calendar year were considered: $\geq 2$ vs. $<2$ drugs; $\geq 4$ vs. $<4$ drugs"; $\geq 6$ vs. $<6$ drugs. In the analysis of determinants the number of drugs was calculated using Algorithm 3; sensitivity analyses were performed with the other two Algorithm and the results 
Citation: Fano V, Chini F, Pezzotti P, Bontempi K (2014) Estimating the Prevalence and the Determinants of Polypharmacy Using Data from a Health Administrative Database: A Comparison of Results Obtained Employing Different Algorithms. Adv Pharmacoepidemiol Drug Saf 3: 151. doi:10.4172/2167-1052.1000151

Page 4 of 7

were consistent (data not shown).

All statistical analyses were performed with the statistical package STATA, version 12 [18].

\section{Results}

A total of 331,923 adults were included in the study population. The demographic characteristics of the study population at the beginning of follow-up are shown in Table 1. Fifty-four percent were females, $31 \%$ were aged 65 or older, $93 \%$ were Italians, $35 \%$ were resident in highly o very highly deprived areas. Median age was 56 years for women and 54 for men. During the study period $17 \%$ of the patients had at least one chronic or severe disease according to Charlson's index definition; the cumulative mortality rate for the 5 years of follow-up was $5.8 \%$.

A total of 24,543,189 prescriptions were retrieved from the Drug Prescription Registry. The percentage of subjects that used at least one drug was $79 \%$ in 2009, and decreased to $75 \%$ in 2012.

Figure 1 illustrates the three algorithms used for the estimation of polypharmacy. Figure 2 illustrates the overall prevalence of polypharmacy (4-digits-ATC) by algorithm; prevalence is also shown for the elderly population and by gender. The percentage of subjects with moderate (i.e., 4-5 drugs) or major (i.e., $6+$ drugs) polypharmacy increased by calendar year varying between $9 \%$ and $11 \%$, and between $6 \%$ and $10 \%$, respectively. Prevalence was higher among elderly (range: $21-23 \%$ and $15-25 \%$ for moderate and major polypharmacy, respectively). Women had higher prevalence rates (range: $9-12 \%$ for moderate and major polypharmacy, respectively) compared to men (range: 9-10\% for moderate and major polypharmacy, respectively). Estimates of polypharmacy showed substantial differences among algorithms. More subjects with moderate polypharmacy were detected in each year, on average, with Algorithm 1 compared with Algorithms 2 and $3(+1.5 \%$ and $+1.1 \% ; \mathrm{p}<0.001)$. The same held true for major polypharmacy $(+3.8 \%$ and $3.0 \% ; \mathrm{p}<0.001)$. When stratifying by gender and age, differences between the algorithms in women and in the elderly were more marked (major polypharmacy: $+10 \%$ for Algorithm 1 and $+8 \%$ vs. Algorithm 2 and $3 ; \mathrm{p}<0.001$ ). Similar results were obtained when analysis was performed using the complete 7-digitsATC-code, although the overall prevalence of major polypharmacy was higher compared to that obtained with the first-4-digit-ATC code (data not shown). As shown in Figure 2, the mobile window method (Algorithm 3) is more sensitive with respect to the fixed windows method (Algorithm 2) in identifying people with polypharmacy.

Table 2 shows the prevalence of polypharmacy in 2011 estimated with Algorithm 3, and stratified by several characteristics of the subjects and of the GPs. Regarding the individual effect: women had a higher prevalence of minor polypharmacy compared to men, while there were no substantial differences for major polypharmacy; polypharmacy (any type) was much more frequent among patients aged $\geq 65$ years, among subjects living in areas with a high deprivation index, and among Italian-born subjects.

Regarding the characteristics of GPs, a slightly lower prevalence of polypharmacy (any type) was observed for women GPs, older GPs and GPs with a higher number of patients.

\begin{tabular}{|c|c|c|c|c|c|c|c|c|c|c|c|}
\hline & & & & igits ATC & & & -digits ATC & & & fference (a) & \\
\hline & & & minor & moderate & major & minor & moderate & major & minor & moderate & major \\
\hline & Gender & & & & & & & & & & \\
\hline & & Women & 21.2 & 10.5 & 7.4 & 20.3 & 10.2 & 8.5 & 0.9 & 0.3 & -1.2 \\
\hline & & Men & 17.6 & 9.7 & 7.5 & 16.7 & 9.4 & 9.4 & 0.8 & 0.2 & -1.9 \\
\hline & Age (years) & & & & & & & & & & \\
\hline & & $34-49$ & 9.4 & 1.9 & 0.6 & 9.3 & 2.1 & 0.9 & 0.1 & -0.2 & -0.3 \\
\hline$\frac{0}{6}$ & & $50-64$ & 24.7 & 10 & 5.7 & 23.6 & 10 & 7.2 & 1.1 & 0 & -1.5 \\
\hline 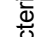 & & $65+$ & 29.4 & 22.4 & 19.3 & 27.7 & 21.1 & 22.5 & 1.7 & 1.2 & -3.2 \\
\hline$\frac{\pi}{\pi}$ & Deprivation index & & & & & & & & & & \\
\hline 등 & & Low & 19.3 & 9.5 & 6.5 & 18.5 & 9.3 & 7.9 & 0.8 & 0.2 & -1.4 \\
\hline ڤొ & & Medium & 19.6 & 10.1 & 7.3 & 18.7 & 9.8 & 8.7 & 0.9 & 0.3 & -1.4 \\
\hline$\frac{\mathscr{D}}{\pi}$ & & High & 19.9 & 11 & 9.1 & 18.9 & 10.6 & 10.8 & 1 & 0.4 & -1.7 \\
\hline & $\mathrm{N}$. of comorbidities & & & & & & & & & & \\
\hline & & None & 19.1 & 8.6 & 5.1 & 18.3 & 8.6 & 6.2 & 0.8 & 0.1 & -1.2 \\
\hline & & $1-2$ & 24.7 & 24.1 & 27.3 & 22.8 & 21.8 & 31.4 & 1.8 & 2.3 & -4.2 \\
\hline & & $3+$ & 15.8 & 23.5 & 50.1 & 14.6 & 20.3 & 54.2 & 1.2 & 3.2 & -4.2 \\
\hline & Nationality & & & & & & & & & & \\
\hline & & Italian & 20 & 10.5 & 7.7 & 19 & 10.2 & 9.3 & 0.9 & 0.3 & -1.5 \\
\hline & & Non-Italian & 15.1 & 6 & 3.8 & 14.5 & 6 & 4.7 & 0.6 & 0 & -0.9 \\
\hline & Gender & & & & & & & & & & \\
\hline & & Women & 18.9 & 9.8 & 7.1 & 18.1 & 9.4 & 8.5 & 0.8 & 0.4 & -1.4 \\
\hline$\stackrel{\mathscr{n}}{=}$ & & Men & 19.8 & 10.3 & 7.5 & 18.9 & 10 & 9 & 0.9 & 0.3 & -1.5 \\
\hline 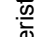 & Age (years) & & & & & & & & & & \\
\hline 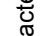 & & $\leq 55$ & 19.2 & 10 & 7.3 & 18.3 & 9.6 & 8.7 & 0.9 & 0.3 & -1.5 \\
\hline$\frac{\pi}{\pi}$ & & $>55$ & 20 & 10.3 & 7.6 & 19.1 & 10 & 9.1 & 0.9 & 0.3 & -1.5 \\
\hline is & Number of patients & & & & & & & & & & \\
\hline 0 & & $<1,000$ & 19.2 & 10 & 7.5 & 18.4 & 9.8 & 8.9 & 0.8 & 0.2 & -1.4 \\
\hline & & $1,000-1,500$ & 20 & 10.4 & 7.6 & 19.1 & 10 & 9.1 & 0.9 & 0.4 & -1.5 \\
\hline & & $>1,500$ & 19.2 & 9.9 & 7.2 & 18.3 & 9.6 & 8.7 & 0.9 & 0.2 & -1.4 \\
\hline
\end{tabular}

† polypharmacy defined according to Algorithm 3

Table 2: Prevalence of polypharmacy for year 2011 according to patients's and GPs' characteristics. Comparisons of estimates using 4-digits vs. 7-digits ATC codes. 
Citation: Fano V, Chini F, Pezzotti P, Bontempi K (2014) Estimating the Prevalence and the Determinants of Polypharmacy Using Data from a Health Administrative Database: A Comparison of Results Obtained Employing Different Algorithms. Adv Pharmacoepidemiol Drug Saf 3: 151. doi:10.4172/2167-1052.1000151

Page 5 of 7

\begin{tabular}{|c|c|c|c|c|c|c|c|c|c|c|}
\hline & & \multicolumn{3}{|c|}{$\geq 2$ vs $<2$ drugs } & \multicolumn{3}{|c|}{$\geq 4$ vs $<4$ drugs } & \multicolumn{3}{|c|}{$\geq 6$ vs $<6$ drugs } \\
\hline & & \multirow[t]{2}{*}{ OR } & \multicolumn{2}{|c|}{$\mathrm{Cl} 95 \%$} & \multirow[t]{2}{*}{ OR } & \multicolumn{2}{|c|}{$\mathrm{Cl} 95 \%$} & \multirow[t]{2}{*}{ OR } & \multicolumn{2}{|c|}{$\mathrm{Cl} 95 \%$} \\
\hline \multirow{18}{*}{ 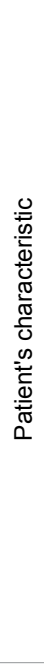 } & Gender & & & & & & & & & \\
\hline & Women & 1.00 & - & - & 1.00 & - & - & 1.00 & - & - \\
\hline & Men & 0.84 & 0.83 & 0.86 & 0.98 & 0.96 & 1.00 & 1.02 & 0.99 & 1.05 \\
\hline & Age (years) & & & & & & & & & \\
\hline & $34-49$ & 1.00 & - & - & 1.00 & - & - & 1.00 & - & - \\
\hline & $50-64$ & 4.71 & 4.61 & 4.81 & 6.49 & 6.24 & 6.75 & 8.48 & 7.86 & 9.13 \\
\hline & $65+$ & 15.38 & 15.04 & 15.74 & 22.22 & 21.40 & 23.07 & 28.15 & 26.20 & 30.25 \\
\hline & Deprivation index & & & & & & & & & \\
\hline & Low & 1.00 & - & - & 1.00 & - & - & 1.00 & - & - \\
\hline & Medium & 1.07 & 1.05 & 1.10 & 1.10 & 1.07 & 1.13 & 1.10 & 1.06 & 1.14 \\
\hline & High & 1.20 & 1.17 & 1.24 & 1.27 & 1.23 & 1.32 & 1.33 & 1.27 & 1.40 \\
\hline & N. of comorbidities & & & & & & & & & \\
\hline & None & 1.00 & - & - & 1.00 & - & - & 1.00 & - & - \\
\hline & $1-2$ & 3.87 & 3.74 & 3.99 & 3.92 & 3.80 & 4.03 & 3.91 & 3.78 & 4.04 \\
\hline & $3+$ & 5.43 & 4.80 & 6.15 & 7.33 & 6.66 & 8.05 & 8.19 & 7.53 & 8.91 \\
\hline & Nationality & & & & & & & & & \\
\hline & Italian & 1.00 & - & - & 1.00 & - & - & 1.00 & - & - \\
\hline & Non-Italian & 0.81 & 0.78 & 0.84 & 0.80 & 0.77 & 0.85 & 0.82 & 0.76 & 0.88 \\
\hline \multirow{10}{*}{ 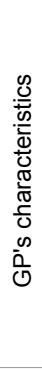 } & Gender & & & & & & & & & \\
\hline & Women & 1.00 & - & - & 1.00 & - & - & 1.00 & - & - \\
\hline & Men & 1.00 & 0.96 & 1.04 & 0.99 & 0.95 & 1.04 & 0.98 & 0.93 & 1.04 \\
\hline & Age (years) & & & & & & & & & \\
\hline & $\leq 55$ & 1.00 & - & - & 1.00 & - & - & 1.00 & - & - \\
\hline & $>55$ & 0.98 & 0.95 & 1.02 & 0.99 & 0.95 & 1.03 & 1.01 & 0.96 & 1.06 \\
\hline & N. of patients & & & & & & & & & \\
\hline & $<1,000$ & 1.00 & - & - & 1.00 & - & - & 1.00 & - & - \\
\hline & $1,000-1,500$ & 1.05 & 1.00 & 1.09 & 1.02 & 0.97 & 1.07 & 1.01 & 0.94 & 1.07 \\
\hline & $1,500+$ & 1.05 & 1.01 & 1.11 & 1.06 & 1.00 & 1.12 & 1.05 & 0.98 & 1.13 \\
\hline
\end{tabular}

† polypharmacy defined according to Algorithm 3 and using up to 4-digits ATC

Table 3: Adjusted Odds Ratios (OR) of polypharmacy† by characteristics of patients and of GPs. Year 2011.

It is of note that prevalence estimates of major polypharmacy were higher using the 7-digit code when compared to the 4-digit code, particularly among older patients and among those with comorbidities. However, the association with the individual and GPs characteristics was similar to that found for the 4-digit- code.

Similar results, in terms of differences, were observed for the other calendar years and when using the other algorithms (data not shown).

Results of multilevel models with respect to year 2011 are reported in Table 3. When comparing subjects with $\geq 2$ vs. $<2$ drugs, we estimated that men had a significant $16 \%$ reduction in polypharmacy compared to women; this reduction diminished to $4 \%$ when comparing $\geq 4$ vs. $<4$ drugs and disappeared when comparing $\geq 6$ vs. $<6$ drugs. Independent of other individual characteristics which were considered, polypharmacy increased significantly with age group and the effect also increased from minor to major polypharmacy. Subjects living in more deprived areas were significantly at higher risk of polypharmacy and this effect increased from 20 to $34 \%$ comparing minor with major polypharmacy. A similar tendency was observed when considering the number of comorbidities. Finally, those born outside Italy were significantly at lower risk of polypharmacy.

No differences were detected for GPs characteristics, except for a slight increase in polypharmacy among GPs having more than 1,500 patients when comparing $\geq 2$ vs $<2$ drugs ( $O R=1.05)$.

We observed a low variation of polypharmacy among individual GPs: the Intra-cluster Correlation Coefficient (ICC) estimated in the model comparing $\geq 2$ vs $<2$ drugs was $1.0 \%$ (95\% Confidence Interval, CI: $0.8-1.2 \%$ ), while in the model comparing $\geq 6$ vs $<6$ drugs the ICC was 1.8\% (95\% CI: 1.4-2.1\%). Similar results were obtained when models were performed using 7-digits-ATC as well as when considering other calendar years (data not shown).

\section{Discussion}

We estimated the prevalence of polypharmacy in a populationbased study of more than 330,000 adult residents in Rome, Italy. We provided estimates testing different approaches, some of which had been in previous studies [10]. In the past drug consumption during a trimester has been considered as a valid proxy to estimate the pointprevalence of polypharmacy $[10,14]$. This proxy was necessary because of the computational difficulties caused by the use of mobile time windows when evaluating multiple prescriptions over defined periods of time. In addition to methods used in previous studies, we have tested a modified algorithm using 90-days mobile windows instead of fixed trimesters. This algorithm provided slightly higher estimates compared to the algorithm with fixed trimesters, and it proved to be more sensitive compared to Algorithm 2 with fixed windows in identifying patients receiving at least 60 days of therapy every three consecutive months.

We have also estimated higher prevalences of polypharmacy than those reported by other studies when using identical methods. Veehof et al. [10] reported a prevalence of $9 \%$ in patients $\geq 65$ years old at the end of follow-up, defining polypharmacy as the use of $\geq 6$ drugs and using the same method as Algorithm 2 with 4-digits-ATC (between 13 and $18 \%$ in our study, Figure 2). A recent study of Italian residents over 
Citation: Fano V, Chini F, Pezzotti P, Bontempi K (2014) Estimating the Prevalence and the Determinants of Polypharmacy Using Data from a Health Administrative Database: A Comparison of Results Obtained Employing Different Algorithms. Adv Pharmacoepidemiol Drug Saf 3: 151. doi:10.4172/2167-1052.1000151

Page 6 of 7

65 years of age, estimated a $11 \%$ prevalence of polypharmacy for the year 2011 - polypharmacy being defined as 10 or more drugs using up to 5-digits ATC - using an algorithm based on the maximum number of drugs registered in a trimester [8]. When applying this method to our dataset we obtained for the year 2011 a $15 \%$ prevalence of 10 or more drugs among the elderly (data not shown).

As expected, Algorithm 1 provided higher estimates compared with the other two, because with this method single drugs prescribed during different periods of the year are considered as overlapping. The other two algorithms seemed more appropriate to identify overlapping drugs; however, Algorithm 3 based on a mobile time window was more sensitive compared to Algorithm 2 with fixed windows (Figure 2). Given that both findings based on 4 and 7-digits codes could be of interest when investigating polypharmacy, both approaches were reported in this study. As expected, higher prevalences of major polypharmacy were obtained when using the 7-digit-ATC code. The choice between the 4 and the 7 -digit code should be addressed according to the context and the objective of the study. In fact, the 4-digit code allows the identification as same therapy of different drugs belonging to the same ATC class, while the 7-digit code permits the discrimination of drugs within the same ATC class when they are simultaneously administered according to a therapeutic protocol (e.g., for HIV treatment). However, apart from any specific indications, it cannot be excluded that the higher prevalence found with 7-digits codes could be partly due to medication duplication (i.e. the prescription of two or more different drugs of the same ATC class).

In considering the results regarding the determinants of polypharmacy, our findings are consistent with other studies conducted both in Italy and in other European countries, although those studies were based on geriatric in-hospital patients' data, excepting a Danish study conducted in the late 90s on the general population [9].

We found that polypharmacy, irrespective of the definition used, was strongly related to age and to the number of comorbidities, as already reported in the literature [7-10]. Vetrano et al. [19] observed an increase in the prevalence of polypharmacy among the elderly when recent hospitalization and/or comorbidities were present. However, they reported a lower prevalence as age and disabilities increased, when the concerns of quality of life and life expectancy outweighs the benefit from multiple therapies. A previous work conducted in our region showed that ATC codes from pharmacy data can provide reliable prevalence estimates of several chronic conditions in the general population, compared with those estimated by hospital information system using list of ICD9-CM diagnosis. It is possible that the presence of other chronic diseases was underestimated in this study, as ambulatory visits are not included in the estimation of patients' diseases; however, this should not have affected the comparisons with similar studies, as the Charlson's comorbidity index is the method most commonly used to estimate the presence of one or more diseases in a group of patients [20].

In our study we also found small changes in the prevalence of polypharmacy related to gender (only for minor polypharmacy), as has been found in other studies conducted in the USA [21] and Europe $[9,22]$. Hofer-Duckelmann [22] ascribed the higher prevalence of polypharmacy found among women above 80 years of age to a variety of factors, including a differing attitude of women compared to men towards the acceptance of drugs, the propensity to consult a physician and the willingness to talk about their problems.

Our finding of a statistically significant increase in polypharmacy in the more deprived areas is consistent with the socioeconomic inequalities already described for this cohort [15] and which has also been reported in the literature with respect to the access to treatments $[23,24]$, drug consumption [21,22] and access to health care [25]. A study of physician utilization in 21 developed countries including Italy, reported that people with higher incomes were significantly more likely to see a specialist than people with lower incomes and, in most countries, with greater frequency [26]. Nevertheless, although the level of deprivation of the area of residence represents a condition of health disadvantage, and although we included into our statistical models other factors related to polypharmacy such as age and comorbidities, it is possible that some residual confounding could affect our results.

The higher prevalence of polypharmacy that we observed among Italian-born citizen, irrespective of all other characteristics considered, may be partly ascribed to the so called "healthy migrant" effect [26], i.e. the hypothesis that first-generation immigrants should be healthier than Italian born-people, and thus have fewer health needs. However, in our study the only information available was the nationality at birth, which did not allow us to distinguish new migrants from those who have lived in Italy for a longer time [27].

It is of note that in our study no particular characteristics of the GPs were found to be associated with the risk of having polypharmacy, except for a slight increase in polypharmacy among GPs having more than 1,500 patients when comparing $\geq 2$ vs $<2$ drugs. Bjerrum and Bergman [28] explored the variation in polypharmacy associated with GPs' characteristics in Denmark, and observed that the number of different drugs prescribed was related to the workload, the number of patients listed per doctor, and the percentage of elderly patients. A cross-sectional study conducted in the Lazio Region, which includes Rome, observed that younger physicians were more likely to prescribe medications, independent of the presence of any chronic condition in their patients; furthermore, female GPs, those practicing in a group and those practicing in urban areas showed higher average drug expenditure [29]. Finally, the observed low variation of patients with polypharmacy between GPs was not surprising; "polypharmacy" itself is not to be considered as a negative nor a positive outcome, but a phenomenon strongly dependent on individual factors, such as age and comorbidities, rather than on GPs' characteristics.

Our analyses were based on retrieval of prescriptions from the regional health service (RHS) for at least 60 days in a year (Algorithm 1) or in a period of 90 days (Algorithms 2 and 3). This and other constraints related to the availability of information imply that our study has some limitations that could affect the results. First, prescription is only a proxy of drug administration; furthermore the date of prescription and the DDDs are only a proxy of the date of starting and of the duration of therapy, respectively. Second, prescriptions from the RHS do not include all the drugs that a person takes; it is possible that in some cases subjects purchased their drugs at their own expense without reimbursement from the RHS and were therefore not recorded in the data sources considered in this study. As health care in Italy is virtually free of charge for any citizen with a chronic condition, it is possible that we are not capturing prescriptions for occasional medications or medications prescribed for minor conditions: as this is more likely to happen in wealthier citizens, this suggests that polypharmacy could be underestimated in this group and the effect of the deprivation index reduced. Furthermore, herbal and over-the-counter products were not included in the data set. A study conducted in Canada raised the question of how the inclusion of other products would affect the definition of polypharmacy; authors identified three patterns of medication usage, considering the use of ATC classified drugs alone or in addition to 
Citation: Fano V, Chini F, Pezzotti P, Bontempi K (2014) Estimating the Prevalence and the Determinants of Polypharmacy Using Data from a Health Administrative Database: A Comparison of Results Obtained Employing Different Algorithms. Adv Pharmacoepidemiol Drug Saf 3: 151. doi:10.4172/2167-1052.1000151

other products, showing that the variation can be ascribed to various socio-demographic characteristics [30]. Third, the choice of 60 days as the minimum length for treatment duration may fail to detect cases of polypharmacy of brief duration, which may be particularly at risk for side effects in acute diseases episodes. Finally, we cannot exclude that potentially interacting drugs were prescribed in different periods which were too close in time to guarantee the wash-out.

In conclusion, our study highlighted that in Italy polypharmacy is an emerging public health issue with increasing prevalence, and that prevalence estimates can largely vary according to the different methods used. We propose a new algorithm which refines the definitions of time windows previously used in the computation of polypharmacy. Moreover, this study contributes to the knowledge of the determinants of polypharmacy, confirming those already known such as age and comorbidities, and exploring other socioeconomic factors related both to patients and to GPs.

Polypharmacy deserves to be further investigated in terms of appropriateness of prescriptions, potential drug-to-drug interaction, and adverse drug events. Further analyses should identify target subgroups in order to properly address prevention programs aimed at reducing unnecessary polypharmacy.

\section{Acknowledgement}

The study is partially supported by the Lazio Region within the independent drug research Program "Regional funds for the Pharmacovigilance 2008-2009". Authors wish to thank Dr Andrea Milano for his help in revising the manuscript.

\section{References}

1. Maggiore RJ, Gross CP, Hurria A (2010) Polypharmacy in older adults with cancer. Oncologist 15: 507-522.

2. Hughes CM (2004) Medication non-adherence in the elderly: how big is the problem? Drugs Aging 21: 793-811.

3. Frazier SC (2005) Health outcomes and polypharmacy in elderly individuals: an integrated literature review. J Gerontol Nurs 31: 4-11.

4. Hilmer SN, Gnjidic D (2009) The effects of polypharmacy in older adults. Clin Pharmacol Ther 85: 86-88.

5. Jensen GL, Friedmann JM, Coleman CD, Smiciklas-Wright H (2001) Screening for hospitalization and nutritional risks among community-dwelling older persons. Am J Clin Nutr 74: 201-205.

6. Gorard DA (2006) Escalating polypharmacy. QJM 99: 797-800.

7. Nobili A, Licata G, Salerno F, Pasina L, Tettamanti M et al. (2011) Polypharmacy, length of hospital stay, and in-hospital mortality among elderly patients in internal medicine wards. The REPOSI study. Eur J Clin Pharmacol 67: 507-519.

8. Onder G, Bonassi S, Abbatecola AM, Folino-Gallo P, Lapi F, et al. (2013) The Geriatrics Working Group of the Italian Medicines Agency (AIFA). High Prevalence of Poor Quality Drug Prescribing in Older Individuals: A Nationwide Report From the Italian Medicines Agency (AIFA). J Gerontol A Biol Sci Med Sci.

9. Bjerrum L, Søgaard J, Hallas J, Kragstrup J (1998) Polypharmacy: correlations with sex, age and drug regimen. A prescription database study. Eur J Clin Pharmacol 54: 197-202.

10. Veehof L, Stewart R, Haaijer-Ruskamp F, Jong BM (2000) The development of polypharmacy. A longitudinal study. Fam Pract 17: 261-267.

11. Hajjar ER, Hanlon JT, Sloane RJ, Lindblad CI, Pieper CF, et al. (2005) Unnecessary drug use in frail older people at hospital discharge. J Am Geriatr Soc 53: 1518-1523.

12. Steinman MA, Landefeld CS, Rosenthal GE, Berthenthal D, Sen S, et al. (2006) Polypharmacy and prescribing quality in older people. J Am Geriatr Soc 54: 1516-1523.

13. Gnjidic D, Le Couteur DG, Pearson SA, McLachlan AJ, Viney R, et al. (2013) High risk prescribing in older adults: prevalence, clinical and economic implications and potential for intervention at the population level. BMC Public Health 13: 115
14. Bjerrum L, Rosholm JU, Hallas J, Kragstrup J (1997) Methods for estimating the occurrence of polypharmacy by means of a prescription database. Eur $\mathrm{J}$ Clin Pharmacol 53: 7-11.

15. Fano V, Pezzotti P, Gnavi R, Bontempi K, Miceli M, et al. (2013) The role of socio-economic factors on prevalence and health outcomes of persons with diabetes in Rome, Italy. Eur J Public Health 23: 991-997.

16. Deyo RA, Cherkin DC, Ciol MA (1992) Adapting a clinical comorbidity index for use with ICD-9-CM administrative databases. J Clin Epidemiol 45: 613-619.

17. CasDR, Desiderio V, Lombardozzi L, Orzella L, Pasquale L, et al. (2009) Roma: IstitutoSuperiore di Sanita; 2011. (Rapportilstisan 11/11).

18. Mardani S, Nasri H2, Hajian S3, Ahmadi A4, Kazemi R1, et al. (2014) Impact of Momordicacharantia extract on kidney function and structure in mice. $J$ Nephropathol 3: 35-40.

19. Vetrano DL, Tosato M, Colloca G, Topinkova E, Fialova D, et al.(2013) Polypharmacy in nursing home residents with severe cognitive impairment Results from the SHELTER Study. Alzheimers Dement 9: 587-593.

20. Chini F, Pezzotti P, Orzella L, Borgia P, Guasticchi G (2011) Can we use the pharmacy data to estimate the prevalence of chronic conditions? a comparison of multiple data sources. BMC Public Health 11: 688

21. Perry BA, Turner LW (2001) A prediction model for polypharmacy: are older, educated women more susceptible to an adverse drug event? J Women Aging 13: $39-51$

22. Hofer-Dückelmann C (2012) Gender and polypharmacotherapy in the elderly: a clinical challenge. Handb Exp Pharmacol : 169-182.

23. Davidsen JR, Sondergaard J, Hallas J, Siersted HC, Knudsen TB, et al. (2011) Impact of socioeconomic status on the use of inhaled corticosteroids in young adult asthmatics. Respir Med 105: 683-690.

24. Hjern A, Haglund B, Rosén M (2001) Socioeconomic differences in use of medical care and antibiotics among schoolchildren in Sweden. Eur J Public Health 11: 280-283.

25. Goddard M, Smith P (2001) Equity of access to health care services: theory and evidence from the UK. Soc Sci Med 53: 1149-1162.

26. van Doorslaer E, Masseria C, Koolman X; OECD Health Equity Research Group (2006) Inequalities in access to medical care by income in developed countries. CMAJ 174: 177-183.

27. Fennelly K (2007) The "healthy migrant" effect. Minn Med 90: 51-53.

28. Bjerrum L, Bergman U (2001) [How many prescription drugs does a general practitioner handle? A prescription database study]. Ugeskr Laeger 163: 33423346.

29. Orzella L, Chini F, Giorgi Rossi P, Borgia P (2010) Physician and patien characteristics associated with prescriptions and costs of drugs in the Lazio region of Italy. Health Policy 95: 236-244.

30. Votova K, Blais R, Penning MJ, Maclure MK (2013) Polypharmacy meets polyherbacy: pharmaceutical, over-the-counter, and natural health product use among Canadian adults. Can J Public Health 104: e222-228. 